\title{
Near-Surface Composition Profiles and the Adhesive Properties of Statistical Copolymer Films Being Model Systems of Pressure Sensitive Adhesive Films
}

\author{
Alexander Diethert, ${ }^{\dagger}$ Yana Peykova,${ }^{\mp}$ Norbert Willenbacher, ${ }^{\ddagger}$ and \\ Peter Müller-Buschbaum* ${ }^{*} \uparrow$
}

Physik-Department LS E13, Technischen Universität München, James-Franck-Strasse 1, 85747 Garching, Germany, and Institut für Mechanische Verfahrenstechnik und Mechanik, Karlsruher Institut für Technologie (KIT),

Kaiserstrasse 12, 76131 Karlsruhe, Germany

\begin{abstract}
Statistical copolymer films that consist of two types of monomers, a sticky and a glassy monomer, are investigated. These model systems of pressure-sensitive adhesive films are probed with X-ray reflectivity and mechanical tack measurements. For the first time, composition profiles along the surface normal in the near-surface region are monitored. The influence of the type of monomers being copolymerized, the monomer ratio, and the sample age on the near-surface composition as well as the resulting adhesive performance of the films are analyzed. The copolymers contain ethylhexyl acrylate as the majority component and styrene, maleic acid anhydride, or methylmethacrylate as a minority component. Regardless of the composition, we find a surface enrichment of one component in all samples. In the case of freshly prepared samples, this enrichment is driven by solubility due to the preparation based on solution casting. The minimization of the surface free energy results in an internal reorganization and the component of the statistical copolymer with the lower surface tension enriches at the free surface. The mechanical behavior is not dominated by the surface but by the surface-near part of the composition profile.
\end{abstract}

KEYWORDS: statistical copolymer $\bullet$ X-ray reflectivity $\bullet$ surface enrichment $\bullet$ pressure sensitive adhesive

\section{INTRODUCTION}

ilms of pressure-sensitive adhesives (PSAs) play an
important role in our everyday life for example in
adhesive foils and tapes, binding materials or stickon notes and in high-tech applications. By applying a slight pressure, a releasable bond to the substrate is formed (1-11). In many PSA applications, a controlled release of the adhesive bond is desired, making use of the ability to undergo several cycles of bonding and detaching. A prominent class of PSAs uses a soft and tacky polyacrylate as its basic component $(12-16)$. With respect to applications, the PSA has to balance conflicting requirements on the viscoelastic properties, the stiffness, and the hardness. For example, on the one hand, good adhesion is desired for the bonding of the PSA to the substrate, but on the other hand, one tries to achieve good cohesion not to avoid flow within the PSA film. To control these parameters, the soft and tacky polyacrylate is often copolymerized with a harder, glassy comonomer (1). Typically two, three, or even more different monomers are combined in a statistical copolymer to balance the different requests of the PSA film. The focus of this

* Corresponding author. Phone: +498928912451. Fax: +498928912473. E-mail muellerb@ph.tum.de

Received for review April 12, 2010 and accepted June 4, 2010

† Technischen Universität München.

* Karlsruher Institut für Technologie

DOI: 10.1021/am100322j

2010 American Chemical Society investigation is on the composition near the film-air interface being completely different from the overall monomer ratio. This region having a thickness on the order of $100 \mathrm{~nm}$ can influence mechanical parameters such as the tack energy or the stress peak during debonding.

The presence of enrichment layers at the polymer-air interface and internal reorganization processes have already been reported for several other classes of polymers, different to statistical copolymers. For example, with neutron reflectometry, the composition profile of thin polymer blend films of deuterated polystyrene (dPS) and poly(para-methylstyrene) (PpMS) was probed (17). Immediately after preparation, the dPS component enriched at the free surface. Because of annealing above the glass-transition temperature of both components and the resulting increase in polymer chain mobility, the blend film reorganized in a way that finally the PpMS component formed the top layer of the blend film. Similar investigations for many other polymer blend films, as for example PS/polybromostyrene (18), PS/ dPS (19), or PS/poly(vinyl methyl ether) (20), show the same phenomenon of surface enrichment of one component of the binary system.

The formation of enrichment layers in block copolymer films caused by the lamellar ordering is also well-documented. The structure of such surface enrichment of one component of the block copolymer at the free interface was studied with many different techniques, among which are grazing incidence small-angle X-ray scattering (21), atomic 
force microscopy (22), neutron reflectometry (23), and transmission electron microscopy (24). It was also shown that the incorporation of nanoparticles in the polymer matrix improved the lamellar structure (25). Furthermore, surfaceinduced ordering was observed in thin films of poly(ethyl acrylate)-polystyrene graft polymers (26). Thus having a one component surface layer is a common behavior for different types of polymer systems but, to the best of our knowledge, it has never been directly observed in statistical copolymers.

In the case of statistical copolymers, only a few indirect hints for surface enrichment were reported. For example, Falsafi et al. measured the surface energies of cross-linked ethylhexyl acrylate-co-acrylic acid elastomers for various acrylic acid contents (27). The surface energy was almost constant, which implies that the surface composition did not vary. The authors concluded from the value of the surface energy that the surface was dominated by methylene groups. However, this investigation could neither quantify the surface composition nor give structural information about regions underneath.

In contrast, theoretical works predicted the presence of surface enrichment causing composition fluctuations in the surface near region of statistical copolymers. For example, Donley and Fredrickson predicted an either oscillatory or monotonic composition profile of random multiblock copolymer melts near surfaces (28). More recently, Klos et al. simulated the composition profile of a two component statistical copolymer $\mathrm{P}(\mathrm{A}$-stat-B) near a selective surface using the bond-fluctuation method (29). The higher the A-concentration at the interface, the higher the B-concentration underneath. Furthermore, conformational rearrangements in the surface layer are predicted.

Because for PSA systems the mechanical behavior is of utmost importance, mechanical tests such as the tack test or the peel test are well-established. Whereas in the tack test, the force causing debonding acts along the surface normal, in a peel test, it is applied under an angle to the surface normal (30-33). From the mechanical measurements, information about the bonding behavior of the PSA is deduced. The influence of characteristic control parameters is determined from tack experiments. One of them is, for example, the level of cross-linking of the polymer chains in the PSA. In a recent study, the authors produced PSA films from latexes (34) in which they activated a cross-linking reaction between the latex particles in the drying stage. A controlled cross-linking density was achieved and, among other parameters, the effect on the stress versus strain curves monitored.

In another study, the tackiness of polystyrene-blockpolybutadiene-block-polystyrene triblock copolymers mixed with hydrogenated aromatic hydrocarbon resins with varying degree of hydrogenation was analyzed $(35,36)$. The authors observed that the probe tack goes through a maximum at that degree of hydrogenation at which the aromatic hydrocarbon shows the most favorable interaction with polybutadiene. As a consequence, the solubility of the polymer can be a suitable parameter in adjusting the adhesive properties.

Furthermore, the ratio between the involved monomers has a high impact on the mechanical properties. In 1993, for example, Kano et al. studied the influence of the acrylic acid content in the statistical copolymer with poly(ethylhexyl acrylate) (PEHA) (37). With increasing acrylic acid content, the storage modulus and the loss modulus increased. In another investigation, Aymonier et al. varied the methylmethacrylate (MMA) content in the statistical copolymer with PEHA (38). With decreasing MMA content, the tack energy increased up to a critical content of $25 \%$. However, a further decrease of the MMA content even reduced the tack energy. When the MMA content was too low, the polymer was not cohesive enough to peel it off the substrate without any stuck residue.

In addition to the allover monomer composition, the internal arrangement of the monomers is of importance for the mechanical behavior. By varying the conditions for the polymerization process of the statistical copolymer P(EHACo-MMA), the authors produced copolymers, which covered a range from polymers with a homogeneous monomer distribution to more and more phase-separating copolymers (39). The observed adhesive properties differed even among such statistical copolymers with the same total monomer ratio. Moreover, Laureau et al. investigated the differences in the tackiness of P(EHA-co-MMA) with different molecular weights and different copolymer composition gradients perpendicular to the sample surface (40). It was observed that the dependence on this so-called copolymer composition profile for low molecular weights was strong and had a reduced influence for higher molecular weights.

In general, all types of mechanical experiments commonly address macroscopic control parameters only. So far, no attempt has been made to relate the mechanical behavior with the microscopic surface structure or with the surfacenear composition profile. In our investigation, we link a characterization of the surface structure and of the surfacenear composition profile with the mechanical information obtained from tack measurements. The structural information is gained from X-ray reflectivity (XRR) measurements with a resolution in the Angstroem-regime $(41-43)$. We focus on statistical copolymers with two components, being a model system for PSAs. We use poly(ethylhexyl acrylate) (PEHA) as the basic component of the PSA. A second comonomer is statistically copolymerized being the minority component. Different glassy commoners, such as styrene (S), maleic acid anhydride (MAA), and methylmethacrylate (MMA) are addressed as minority components.

We observe that regardless of the investigated sample, for all PSA films, one of the two components of the statistical copolymer is enriched at the free surface. The shape of the composition profile in the surface-near region exhibits a damped oscillatory shape. The homogeneous bulk material is reached in a depth on the order of $100 \mathrm{~nm}$. The type of component being enriched at the surface strongly depends on the choice of the minority component. The adhesive 
properties are driven by the composition profile in the nearsurface region. Thus the mechanical behavior depends on the individual copolymer composition.

Moreover, we investigate the aging of these model PSA films. We observe that with increasing age of the sample, the composition profile changes at the surface and in the surface-near region. Because of an internal reorganization process minimizing the surface energy, a freshly prepared PSA film has a different surface composition than a film that is a few weeks old. However, these internal reorganizations have no significant influence on the probed tackiness, which results from basically unchanged integral composition in the surface-near region.

This article has the following structure: After a description of the investigated samples and a brief introduction to the main experimental methods XRR and mechanical tack test, the composition profiles and their influencing factors as well as the consequences on the adhesive behavior are presented and discussed. The article concludes with a summary of the results and a short outlook.

\section{EXPERIMENTAL SECTION}

2.1. Sample Preparation. The polymer films were prepared on microscope slides (MENZEL, $76 \mathrm{~mm} \times 26 \mathrm{~mm} \times 1 \mathrm{~mm}$ ), which were chemically precleaned. For cleaning, the substrates remained $15 \mathrm{~min}$ in a bath consisting of $70 \mathrm{~mL}$ of $\mathrm{H}_{2} \mathrm{O}_{2}, 165$ $\mathrm{mL}$ of $\mathrm{H}_{2} \mathrm{SO}_{4}$, and $45 \mathrm{~mL}$ of deionized water at a temperature of $80{ }^{\circ} \mathrm{C}$. To remove any residues of the bath, we rinsed the substrates in deionized water and dried them with compressed nitrogen afterward (44).

Directly after being cleaned, the substrates were coated with $1 \mathrm{~mL}$ of a toluene solution in which the statistical copolymers were dissolved. A waiting time of about $24 \mathrm{~h}$ under ambient conditions turned out to be appropriate for the solvent to be fully evaporated. In this time, the samples were kept in a closed box to avoid UV-degradation. The thickness of all investigated samples is approximately $50 \mu \mathrm{m}$, which is controlled by the solution concentration and also cross-checked by weight measurements. For the aging experiments, the samples were again stored in a closed cabinet at a temperature of $20^{\circ} \mathrm{C}$, which was kept constant by air-conditioning.

In general, each statistical copolymer in this investigation consists of two kinds of monomers, a sticky component and a glassy component. The sticky monomer ethylhexyl acrylate (EHA) is the majority component of the copolymer. To investigate the influence of the chemical composition of the minority component, we use three different glassy monomers. Representing the minority component in the copolymer, these are styrene (S), maleic acid anhydride (MAA), and methylmethacrylate (MMA). The selected monomer composition is $90 \%$ EHA and $10 \%$ of the glassy monomer and the molecular weights are $M_{\mathrm{w}}=182 \mathrm{k}$ for P(EHA-stat-S), $M_{\mathrm{w}}=187 \mathrm{k}$ for P(EHA-stat-MAA), and $M_{\mathrm{w}}=165 \mathrm{k}$ for P(EHA-stat-MMA). Moreover, a second type of $\mathrm{P}(\mathrm{EHA}$-stat-MMA) is used with an altered monomer ratio of $80 \%$ EHA and $20 \%$ MMA and $M_{\mathrm{w}}=248 \mathrm{k}$. This statistical copolymer, which is used in the aging experiment, is referred to as P(EHA-stat-20MMA)

All used statistical copolymers were polymerized with the radical solution polymerization technique and thus show broad molecular weight distributions, which is typical for adhesive applications. The model polymers were synthesized in a semibatch procedure in iso-butanol at $100{ }^{\circ} \mathrm{C}$ and $70 \%$ solids content with a peroxide starter.

2.2. X-ray Reflectivity (XRR). All XRR experiments were performed with a "Siemens D5000 Diffraktometer" under room temperature. The wavelength of the X-rays was $\lambda=0.154 \mathrm{~nm}$, which corresponds to the $\mathrm{Cu}-\mathrm{K}_{\alpha}$ line, and the beam width was set to $12 \mathrm{~mm}$. To minimize the beam divergence, we collimated it with a tantalum knife edge and a slit system. The reflected $\mathrm{X}$-rays were detected with a scintillation counter, in front of which a graphite monochromator was installed in order to filter the $\mathrm{Cu}-\mathrm{K}_{\beta}$ line. To avoid detector saturation for small reflection angles, and thus wrong counting rates, we used an absorber, which reduces the intensity by a factor of around 100 .

For an incident angle $\theta$, the detector was positioned at an angle of $2 \theta$ with respect to the incoming beam. The angular range was chosen to $0^{\circ}<2 \theta<7^{\circ}$ with a maximum resolution of $0.01^{\circ}$. An accumulation time of approximately $12 \mathrm{~h}$ turned out to be appropriate to get sufficient statistics. For a pronounced representation of the features in the reflectivity curves, we plot $\left(I\left(q_{z}\right)-b\right) q_{z}{ }^{4}$ as a function of the scattering vector $q_{z}=$ $4 \pi \sin (\theta) / \lambda$. In this so-called Fresnel-normalized representation, $I\left(q_{z}\right)$ is the reflected intensity normalized to 1 and $b$ is the background, which is assumed to be constant.

The data analysis was performed with the reflectivity simulation and analysis tool Parrat32 (45). With this software, we calculated the reflectivity of an assumed refractive index profile by dividing it into $1 \AA$ thick slices that have no roughness via the Parratt algorithm (41). Each profile can be modeled by approximately 10 parameters. The calculated reflectivity is compared to the measured data, the assumed refractive index profile is then modified, and again, the reflectivity is calculated. Iteratively, a fit to the data is achieved and a refractive index profile is obtained.

2.3. Mechanical Tack Test. To estimate the contribution of the monomers S, MAA, and MMA to the adhesion of the PSAs as well as to determine the change of the adhesive properties in time, we carried out tack measurements with a flat cylindrical punch. The used experimental setup is a "Texture Analyzer TA.XTplus" (Stable Micro System, UK) equipped with an extremely stiff quartz load cell (stiffness $40 \mathrm{~N} / \mu \mathrm{m}$, force range $\pm 500 \mathrm{~N}$, threshold $1 \mathrm{mN}$ ). The punch was prepared out of stainless steel polished with abrasive paper. The average roughness is determined with scanning probe microscopy and is found to be $R_{\mathrm{a}}=5.1 \mathrm{~nm}$. During the tack test, the glass slide coated with the polymer film is positioned on a vacuum table. The punch contacts the PSAs film with a contact force of $10 \mathrm{~N}$; after a contact time of $1 \mathrm{~s}$, it is withdrawn with a constant speed of $0.1 \mathrm{~mm} / \mathrm{s}$. After each measurement, the surface of the probe is cleaned with acetone in order to remove dust or any PSA residues. The test is performed at room temperature and repeated serveal times to gain sufficient statistics. Subsequent tack experiments are always performed on fresh PSA film surfaces. During the debonding process the video images were obtained with a high-speed camera KL MB-Kit 1 M1 (Mikrotron $\mathrm{GmbH}$, Germany) used in combination with zoom $90^{\circ} \mathrm{KL}-\mathrm{Z} 6$ and cold light source KL3000B. This setup allows to record 124 frames/s with $1280 \times 1024$ pixels (1 pixel is approx. $5 \mu \mathrm{m}$ ) resolution. Force-time curves were synchronized with the video sequences in such a way that the first contact of the probe with the sample in the force curve corresponds to the image showing the first contact. The videos were quantitatively analyzed using Visiometrics Image Processing System software (Prof. Dr. Stephan Neser, University Darmstadt) in order to determine the true contact area and the number and growth rate of individual cavities (61)

2.4. Optical Microscopy. The films were observed with a "Zeiss Axiotech $25 \mathrm{H}$ " optical microscope using magnifications between 5 and $100 \times$. The micrographs were recorded with a "Hitachi KP-D50" CCD camera. 


\section{RESULTS AND DISCUSSION}

The near-surface refractive index profile is measured with X-ray reflectivity (XRR). XRR has many advantages: it gives a resolution in the Ångstroem-regime; the whole refractive index profile can be extracted out of one measurement; it is nondestructive; no special sample preparation is required; and one gets averaged information over the whole illuminated sample area (41-43).

Because we investigate polymer films with a thickness of $50 \mu \mathrm{m}$, the X-ray beam does not penetrate the whole polymer film and thus does not reach the glass substrate (46). As a consequence, we obtain no total reflection from the glass substrate but only total external reflection from the polymer film surface. This allows us to achieve the highest possible sensitivity of the XRR measurements with respect to the film composition close to the sample surface. In case of thinner polymer films, with film thicknesses of $20 \mu \mathrm{m}$ or less, the X-ray beam is totally reflected from the substrate as well, which results in the presence of a second critical angle (of glass) in the XRR data. The modeling of such data is more challenging and surface enrichment layers are more difficult to be separated from enrichment layers at the substrate. Anyhow, a variation in the film thickness was beyond the scope of this investigation.

The chemical composition of the polymer film can be calculated out of the refractive index profile $\delta(z)$, where $\delta$ is the real part of the complex refractive index $n=1-\delta+i \beta$ and $z$ is the distance from the sample surface. $\delta$ is proportional to the electron density and thus depends on the mass density of the polymer, the number of electrons in the respective monomers, and the corresponding dispersion correction factors in dipole approximation of the atom form factor $(43,47)$.

The values for the involved homopolymers related to the statistical copolymers are $\delta(\mathrm{PEHA})=3.21 \times 10^{-6}, \delta(\mathrm{PS})=$ $3.62 \times 10^{-6}, \delta(\mathrm{PMAA})=4.25 \times 10^{-6}$, and $\delta(\mathrm{PMMA})=4.06$ $\times 10^{-6}$. The high contrast between $\delta$ (PEHA) and $\delta$ of the respective minority component makes it easy to distinguish between the two components of each investigated statistical copolymer. The average refractive indices of the statistical copolymers are calculated by weighting the refractive indices of the respective homopolymers by their composition ratios: $\delta(\mathrm{P}($ EHA-stat-S $))=3.25 \times 10^{-6}, \delta(\mathrm{P}($ EHA-stat-MAA $))=3.31$ $\times 10^{-6}, \delta(\mathrm{P}($ EHA-stat-MMA $))=3.29 \times 10^{-6}$ and $\delta(\mathrm{P}(\mathrm{EHA}-$ stat-20MMA)) $=3.37 \times 10^{-6}$.

Once a refractive index profile $\delta(z)$ of a film consisting of two components is extracted out of the reflectivity data, it is converted into a composition profile by applying the rule of three and using the above values of the real part of the refractive index $\delta$. This becomes possible because all investigated PSA samples consist of statistical copolymers with two components with known refractive indices.

3.1. Composition Profiles of Freshly Prepared Samples. To rule out the influence of aging, we first address the composition profiles of freshly prepared films of P(EHA-stat-S), P(EHA-stat-MAA) and P(EHA-stat-MMA). Panels a, $\mathrm{C}$, and e in Figure 1 show the Fresnel-normalized
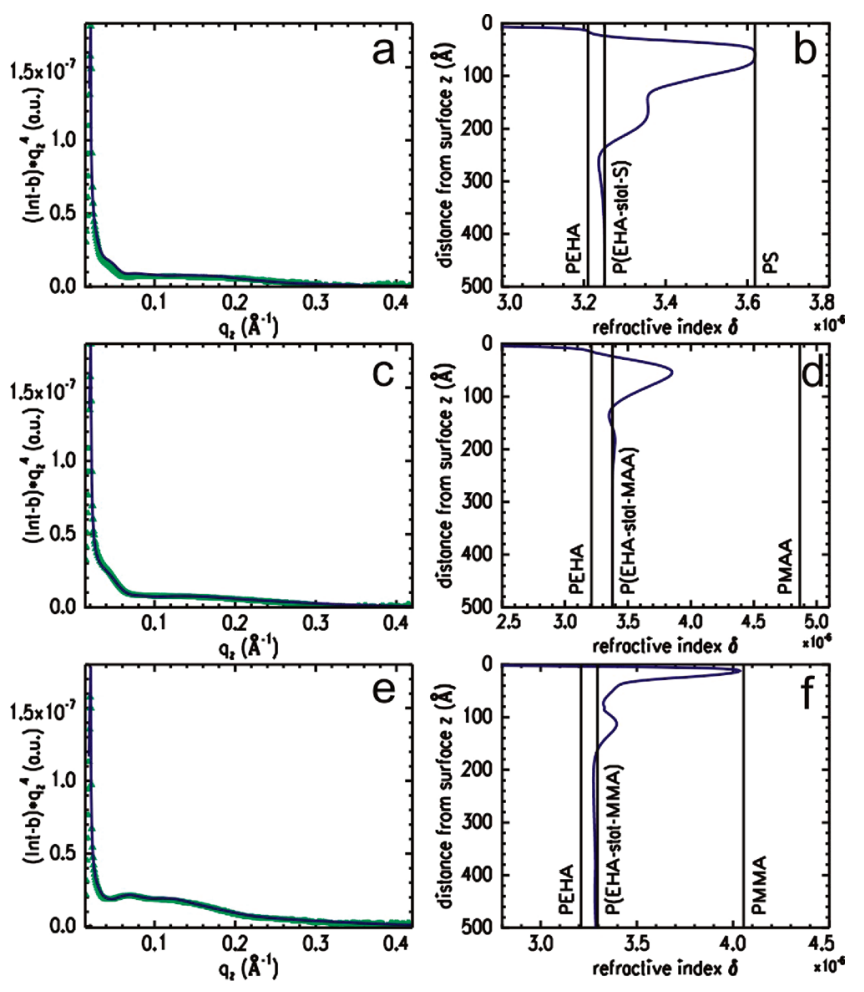

FIGURE 1. Left: Measured XRR data (symbols) of freshly prepared (a) P(EHA-stat-S), (c) P(EHA-stat-MAA), and (e) P(EHA-stat-MMA) films and respective fits to the data (solid lines) in Fresnel-normalized representation. Right: $(b, d, f)$ Corresponding refractive index profiles of the shown fits. The vertical lines mark the values of the refractive index of the statistical copolymer and the related two homopolymers as shown by the labels.

data (triangles) with the corresponding fits (solid lines). The respective refractive index profiles are depicted in panels $b$, $\mathrm{d}$, and $\mathrm{f}$ in Figure 1. The black solid lines are positioned at the values of the refractive indices of the involved (homo)polymers and the depth $z=0$ denotes the sample surface.

The modulations in the reflectivity curves in the range of $0.02 \AA^{-1}<q_{z}<0.3 \AA^{-1}$ already indicate a heterogeneous refractive index profile and thus an enrichment of one component of the statistical copolymer in the near-surface part of the adhesive film. Detailed information is extracted from the refractive index profiles (right column of Figure 1). For these polymers, the enrichment takes place only in the region between the sample surface and a depth of approximately $z=40 \mathrm{~nm}$. For larger depths $(z>40 \mathrm{~nm})$, we find homogeneous material with the calculated average refractive index of the statistical copolymer. This has already been indicated by former small-angle X-ray scattering experiments where nanophase segregation in the bulk could not be detected.

P(EHA-stat-S) and P(EHA-stat-MAA) show a very similar behavior. For both polymers, the majority component PEHA is enriched at the sample surface which is identified from the shoulders in the refractive index profile at the value of PEHA. Underneath this enrichment layer of PEHA an enrichment of the minority components is observed, which reach their maxima at a shallow depth of $z=5.9 \mathrm{~nm}$ for P(EHAstat-S) (Figure $1 \mathrm{~b})$ and at $z=5.4 \mathrm{~nm}$ for P(EHA-stat-MAA) 
(see Figure 1d). At these maximum positions, the monomer ratios are $100 \%$ PS and $61.3 \%$ PMAA, respectively. For larger distances from the film surface, the refractive indices oscillate into the average refractive index of the corresponding statistical copolymer.

In contrast to these two copolymers, it is the minority component PMMA that is enriched in P(EHA-stat-MMA) at the film surface (see Figure $1 \mathrm{f}$ ). It has a maximum contribution of $97.6 \%$ in a depth of $z=1.2 \mathrm{~nm}$. Again, for larger distances from the surface, the refractive index converges in an oscillating way to the refractive index of the copolymer.

It has to be noted that on the basis of a freely jointed polymer chain, the calculated radii of gyration of the copolymers used are between $R_{\mathrm{g}}=8.4$ and $11.2 \mathrm{~nm}$. As a consequence, the thicknesses of the observed enrichment layers at the polymer-air interface are in the sub- $R_{\mathrm{g}}$ regime. More detailed information concerning chain conformation, however, cannot be deduced from the XRR data.

The explanation to which sort of monomer is enriched at the surface $(z<2 \mathrm{~nm})$ of the freshly prepared samples can be found in the different solubilities of the involved homopolymers in toluene and the used preparation technique solution casting. Because the solvent evaporates through the surface, it can transport material from the bulk to the surface during evaporation. This transport is a selective process. Polymer chains with a higher ratio of that monomer, which has the better solubility, are preferentially brought to the sample surface. The solubility parameters available in literature (48) are $\Delta$ (toluene) $=18.2 \mathrm{MPa}^{0.5}$, $\Delta(\mathrm{PEHA})=18.37 \mathrm{MPa}^{0.5}, \Delta(\mathrm{PMMA})=18.27 \mathrm{MPa}^{0.5}$, and $\Delta(\mathrm{PS})=22.47 \mathrm{MPa}^{0.5}$. These values strongly support the described mechanism. Such solubility-driven morphologies were reported in the case of polymer blend films $(49,50)$ and block copolymer films $(51,52)$ as well, and it is therefore plausible that statistical copolymers exhibit the same behavior.

The composition of the subsurface region $(2 \mathrm{~nm}<Z<40$ $\mathrm{nm}$ ) is a consequence of the solubility driven enrichment at the surface. The latter is a constraint on the composition of the rest of the film. Most polymer chains in the film are composed of both types of monomers. The choice of one monomer to enrich at the surface results in a selective segregation and a deformation of the polymer chains in a way that the constraint is fulfilled. As a consequence, there is a lack of the surface-enriched monomer in the subsurface region and the other type of monomer dominates. Going deeper inside the film the same effect applies again and the dominating monomer changes once more resulting in an oscillatory profile. In contrast to block copolymers, the amplitude decreases with the number of oscillations until they are no longer detectable and homogeneous bulk material is reached $(z>40 \mathrm{~nm})$. In the bulk, no constraints act on the system and thus no modulations of the composition are present. The bulk shows simply the mean composition of the statistical copolymer.

It has to be noted that the integral over the whole nearsurface composition profile is not equal to the total monomer

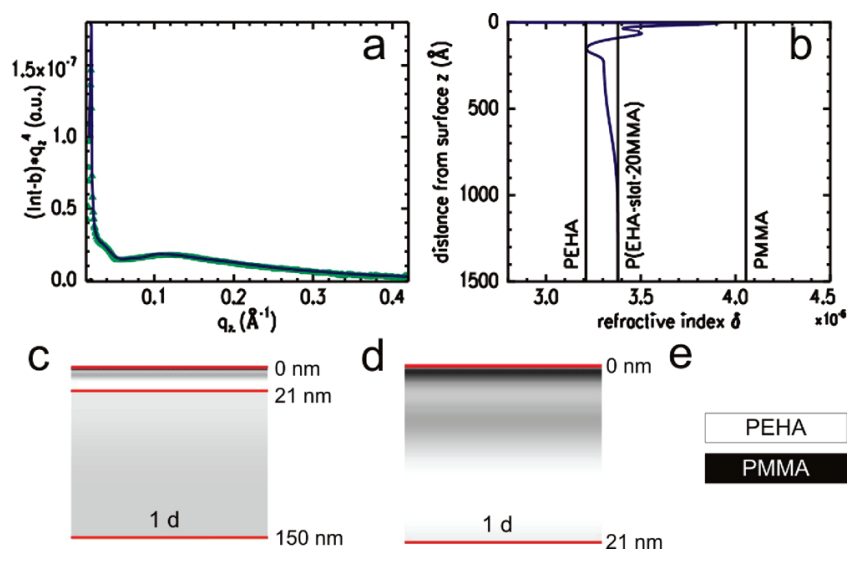

FIGURE 2. (a) Measured XRR data (symbols) of a freshly prepared $\mathrm{P}$ (EHA-stat-20MMA) film and respective fit to the data (solid line) in Fresnel-normalized representation, (b) corresponding refractive index profile, (c) black and white coded composition profile calculated from the refractive index profile, (d) zoom into the surface near region of the composition profile, and (e) color code used in the composition profiles.

composition ratio because mass conservation does not apply for the limited probed region close to the film surface. As described above, by purpose we have no access to information about the composition profile near the polymer-glass interface with the XRR setup used.

Because of the very large number of polymer chains, which are composed in the adhesive film of $50 \mu \mathrm{m}$ thickness, polymer chains of any possible composition out of the two copolymerized monomers can be found in the bulk. Therefore, for freshly prepared samples, the type of monomer enriched close to the film surface should only depend on the choice of the solvent and the monomers in the statistical copolymer. This is supported by the refractive index profile of a freshly prepared P(EHA-stat-20MMA) film (see Figure $2 b$ ) in which the amount of copolymerized MMA is increased by a factor of 2 . The measurement was performed one day after the dissolved polymer was coated on the substrate, which is referred to as the sample age. The profile looks very similar to that of the copolymer with $10 \%$ MMA, except that homogeneous bulk material is reached at a depth of approximately $z=100 \mathrm{~nm}$. Furthermore the maximum PMMA concentration is $77.0 \%$ and it is reached for a depth of $z=$ $0.9 \mathrm{~nm}$. Additionally, there is a more pronounced PEHAdominated region compared to P(EHA-stat-MMA): for a depth $z=15.3 \mathrm{~nm}$, we find $99.6 \%$ PEHA.

In an alternative presentation, the refractive index profile is converted into a chemical composition profile. The latter is coded in black and white for better illustration (see Figure $2 \mathrm{c}-\mathrm{e}$ ). The horizontal lines mark the depths $z=0,21$, and $150 \mathrm{~nm}$. The zoom into the surface-near region of the film (see Figure 2d) clearly shows a strong enrichment of PMMA at the surface and a second, weaker enrichment layer underneath.

3.2. Composition Profiles As a Function of Time. Because the composition profile of a freshly prepared sample is influenced by the different solubilities of the involved homopolymers the resulting profile is not necessarily an equilibrium structure. After the extractable solvent 


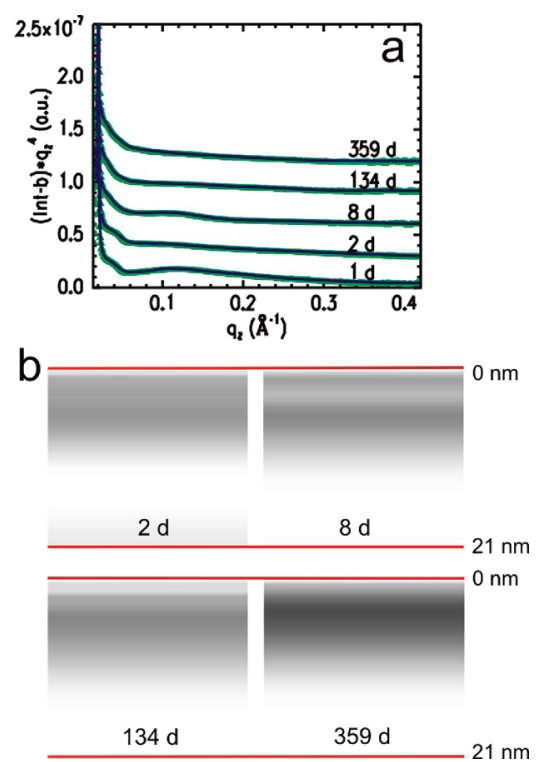

FIGURE 3. (a) Measured XRR data (symbols) of a time series of $\mathrm{P}$ (EHA-stat-20MMA) films and respective fits to the data (solid lines) in Fresnel-normalized representation. The data are shifted along the intensity axis for clarity of the presentation. (b) Composition profiles calculated from the refractive index profiles using the color-code defined in Figure $2 \mathrm{e}$.

has evaporated an internal reorganization process of the film, which we also call aging of the film, can occur to allow for a relaxation toward an equilibrium structure. Because of the low glass-transition temperature of the majority component PEHA of $-50{ }^{\circ} \mathrm{C}(48)$, the system is rather mobile. Non-extractable, residual solvent is not expected in the probed near-surface region; most of it is trapped at the polymer-glass interface as shown in ref 53 . To investigate the aging process, we performed XRR measurements with films of P(EHA-stat-20MMA) of different sample ages.

In Figure 3a, the reflectivity curves of films measured 1, $2,8,134$, and 359 days after casting the solution on the substrate are depicted. Again, a good agreement between measurement (symbols) and the corresponding fits (solid lines) is achieved. The black- and white-coded (see code in Figure 2e) composition profiles in the region between the sample surface $(z=0)$ and a depth of $z=21 \mathrm{~nm}$ are shown in Figure $3 b$.

During the whole investigated time the samples exhibit a heterogeneous composition profile for a narrow nearsurface region $(0 \mathrm{~nm}<z<21 \mathrm{~nm})$. For a depth of $z=21 \mathrm{~nm}$ we find a zone of almost pure PEHA. For depths $z>21 \mathrm{~nm}$, the composition converges monotonically to the average monomer composition of $80 \%$ EHA and $20 \%$ MMA, which is not shown in Figure $3 \mathrm{~b}$ for clarity. This part of the composition profile looks very similar to the one of the freshly prepared sample which is shown in Figure 2c. The bulk material with the nominal monomer ratio is reached in a depth of about $100 \mathrm{~nm}$ for all investigated sample ages.

In more detail, the aging process comprises several stages: After 2 days, the two pronounced layers of PMMA of the freshly prepared sample collapse almost completely. Subsequently, more and more PEHA enriches at the surface and after 134 days, we find an enrichment layer of PEHA at the film surface. Underneath (between $3 \mathrm{~nm}<Z<11 \mathrm{~nm}$ ), there is a zone with an increased content of PMMA but no pronounced layering. The maximum PMMA content is $45.8 \%$, which is reached at a depth of $z=5.8 \mathrm{~nm}$.

Compared to the state after 134 days, the shape of the composition profile after almost 1 year has not changed much, which is also indicated by the strong similarity of the corresponding reflectivity curves. Especially the enrichment of PEHA at the surface is preserved. A difference arises in the zone of increased PMMA content. The PMMA-dominated volume has become smaller but more pronounced and it is shifted a little toward the sample surface. The maximum contribution of PMMA has risen to $65.5 \%$ in a depth of $z=$ $4.4 \mathrm{~nm}$. An explanation of this increase in PMMA concentration could be the self-attraction of the MMA monomers in this region.

In summary, the aging behavior is characterized by a change from the solubility-driven enrichment of PMMA to an enrichment of PEHA. The literature values of the surface tensions of the involved homopolymers are $\gamma(\mathrm{PEHA})=30$ $\mathrm{mN} / \mathrm{m}$ and $\gamma($ PMMA $)=38.5 \mathrm{mN} / \mathrm{m}(54,55)$. Thus an equilibrium structure is installed by minimizing the surface free energy. The subsurface region is structured according to the above explanation and, in addition to that, the attraction of equal monomers plays an important role in the aging process.

It has to be noted that the time scale of the reorganization is slow as compared to the reported chain diffusivities $(56,57)$. For bulk polystyrene annealed above the glasstransition temperature it is reported to be $11.2 \times 10^{-13} \mathrm{~cm}^{2} / \mathrm{s}$ (57) and for thin films it is even lower. To get an idea about the corresponding relaxation times in such a system we assume Brownian motion and calculate approximately $100 \mathrm{~s}$ for a root-mean-square displacement of $150 \mathrm{~nm}$. However, one has to consider the lower temperature of our experiment and the fact that it is not one single chain moving but all chains at least in the near-surface region causing the reorganization process.

To confirm that the minimization of the surface free energy is the origin of reorganization, the aging of P(EHAstat-S) is also investigated. Because PS has a surface tension of $\gamma(\mathrm{PS})=38 \mathrm{mN} / \mathrm{m}$ (58), which is higher than that of PEHA, and because for the freshly prepared sample PEHA is already enriched at the film surface (see Figure 1b), no major morphological rearrangement of components is expected. With XRR a film of P(EHA-stat-S) is measured after 25 days. Figure $4 \mathrm{a}$ shows the reflectivity curve (triangles) with the corresponding fit (solid line). As expected, the clear shoulder at the value of the refractive index of PEHA at the film surface (see Figure $4 \mathrm{~b}$ ) is preserved. In the region underneath, only minor changes occur: PS reaches a maximum contribution of $65.6 \%$ at a depth of $z=5.5 \mathrm{~nm}$. In contrast to the freshly prepared sample, we find a layer of almost pure PEHA for a depth of $15 \mathrm{~nm}<Z<35 \mathrm{~nm}$, which is again attributed to the self-attraction of EHA monomers. The homogeneous bulk material is reached approximately for a depth $z=65 \mathrm{~nm}$. 

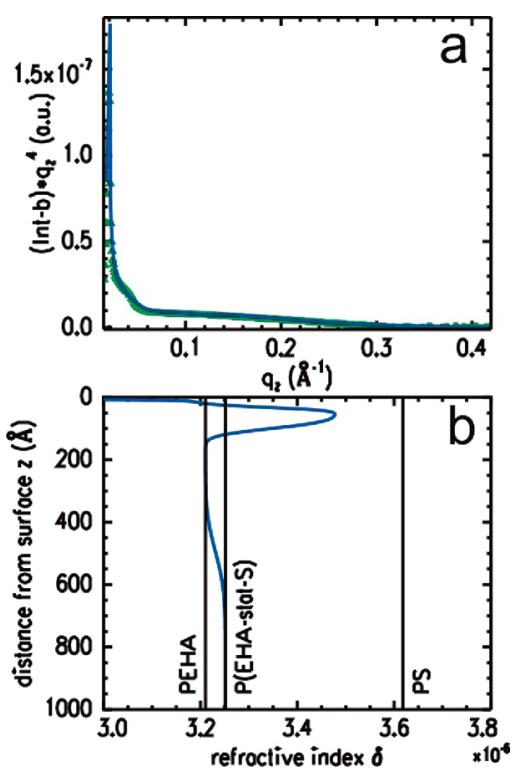

FIGURE 4. (a) Measured XRR data (symbols) of an aged P(EHA-statS) film and respective fit to the data (solid line) in Fresnel-normalized representation and (b) corresponding refractive index profile.
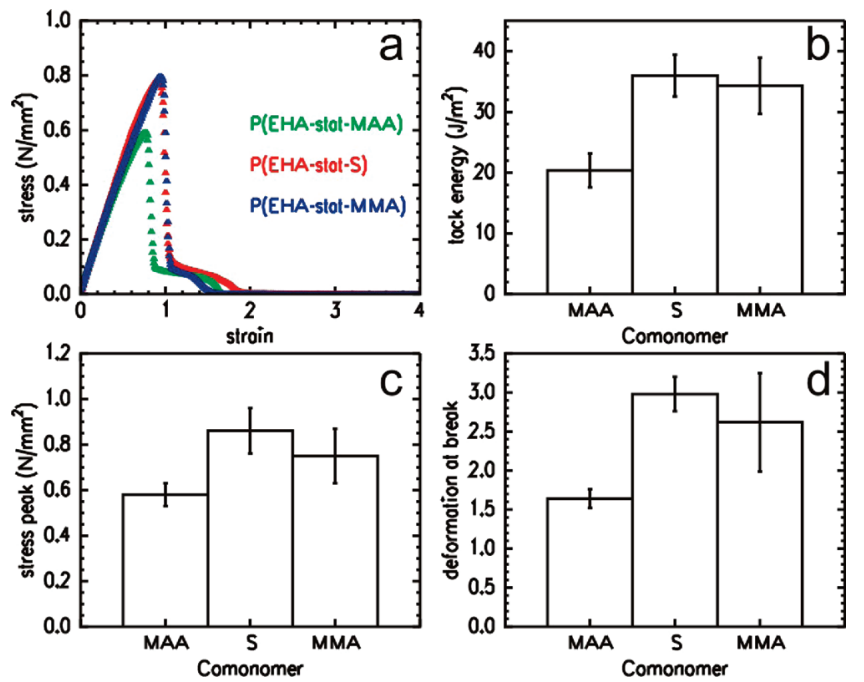

FIGURE 5. (a) Representative stress versus strain curves of films of P(EHA-stat-MAA), P(EHA-stat-S), and P(EHA-stat-MMA), (b) tack energy, (c) stress peak, and (d) deformation at break as a function of the copolymerized minority component.

3.3. Adhesive Properties. To examine whether the recorded composition profiles influence the adhesive properties, we performed mechanical tack tests on freshly prepared samples with varying minority components as well as on an aging series of P(EHA-stat-20MMA).

Figure 5 a shows representative examples of recorded tack curves of freshly prepared films consisting of $90 \%$ EHA and $10 \%$ of a glassy monomer (see sample names in Figure 5a). The dimensionless strain is the ratio between the displacement of the punch and the film thickness and the stress is the force normalized to the punch area (59). The integrals of these functions, the so-called tack energy (see Figure $5 b$ ), show similar values for P(EHA-stat-S) and P(EHAstat-MMA) and a significantly lower value for P(EHA-statMAA). The same holds for other characteristic parameters like the stress peak (Figure 5c) or the strain at which the adhesive force reaches zero (Figure $5 \mathrm{~d}$ ), here referred to as deformation at break (60).

These differences cannot be attributed to viscoelastic properties of the bulk polymer film. The $G^{\prime}$ and $G^{\prime \prime}$ values especially for the samples with MAA and MMA as comonomer are almost identical in a wide frequency range. Furthermore, the plateau of the stress after the initial peak and the length of this stress plateau, which are both controlled by the stretching of lamellae/fibrils and hence by the viscoelastic properties of the polymer are also very similar. Therefore, the low tack of the MAA sample has to be treated as an interfacial phenomenon. This is further supported by the huge difference in the initial stress peak resulting from the differences in the formation and growth of cavities (61) and thus by the interfacial wetting and adhesion properties of the polymer.

This implies that it is the near-surface composition which is responsible for the observed mechanical behavior. However, for a comparison between measured structure and mechanical observations, the surface roughness of the used punch has to be taken into account. The punch has no ideal flat surface that contacts only the films surface, but its surface roughness causes the punch to probe a surface-near part of the film. As a consequence, no agreement between the determined surface component and the tack is observed. For a better comparison, the composition profiles determined with XRR (see right column of Figure 1) are integrated over the punch roughness. From this integration, a total minority component content in the region $0<Z<5.1 \mathrm{~nm}$ is determined to be $42.1 \%$ PMAA for P(EHA-stat-MAA), $66.4 \%$ PS for P(EHA-stat-S), and $49.8 \%$ PMMA for P(EHA-statMMA).

Thus the reduced PMAA content in the surface-near region of the $\mathrm{P}$ (EHA-stat-MAA) film results in a different cavitaion process, causing the lower performance of the $\mathrm{P}$ (EHA-stat-MAA) in the tack test. This becomes visible in Figure 6a, where the number of cavities is plotted versus the strain normalized to the strain peak position. A sharp increase in the number of cavities is obtained for normalized strain values of approximately 0.8 . Shortly before the stress approaches its maximum also the maximum number of cavities is reached for all samples. Their total number is highest for P(EHA-stat-MAA), which is also indicated by the microscopy images in the Figures $6 b-d$. Therefore, we find a correlation between the mechanical performance as accessible in a tack test and the near-surface composition profile as probed with XRR in case of the series varying the minority comonomer.

A similar correlation is observed in the aging experiment. Tack measurements on films of P(EHA-stat-20MMA) have been performed 2, 6, 17, 29, and 49 days after casting the film on the glass substrate (see Figure 7). Within the achieved accuracy of the tack experiment no change in the adhesive properties is detected. Tack energy, stress peak and deformation at break are constant within the experimental errors. Looking at the composition profiles extracted from the XRR data, the surface structure of the P(EHA-stat-20MMA) films 

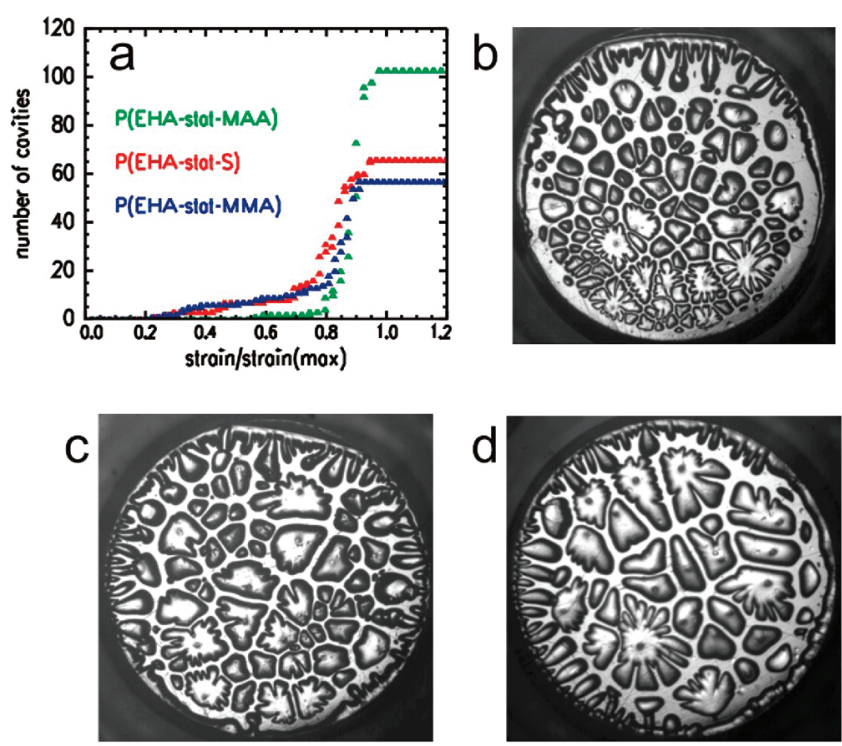

FIGURE 6. (a) The number of cavities as a function of the strain normalized to the stress peak position for $\mathrm{P}$ (EHA-stat-MAA), $\mathrm{P}$ (EHAstat-S), and P(EHA-stat-MMA), and representative microscopy images of the contact area between the punch and the PSA during the tack experiment for (b) P(EHA-stat-MAA), (c) P(EHA-stat-S), and (d) P(EHAstat-MMA).
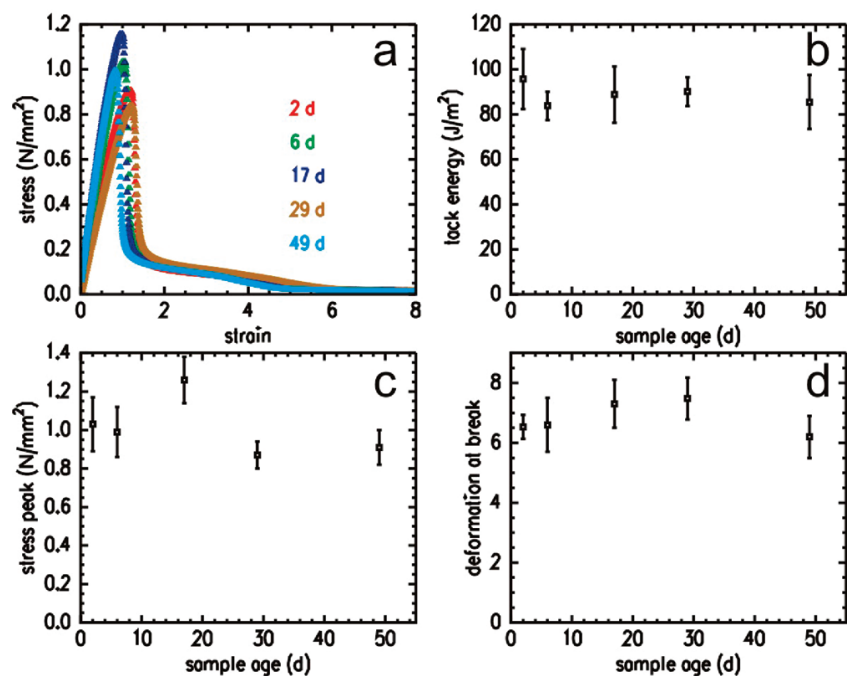

FIGURE 7. (a) Representative stress versus strain curves of P(EHAstat-20MMA) films of different sample age (see values within figure), (b) tack energy, (c) stress peak, and (d) deformation at break as a function of time.

changes strongly (see Figure 3). However, the PMMA content integrated over the punch roughness varies only very little (between 31.7 and $33.3 \%$ ) for the probed time interval. Therefore, again, the near-surface composition dominates the tack test.

The reduced PMMA mobility in the P(EHA-stat-20MMA) films at room temperature, because of a glass-transition temperature of $105^{\circ} \mathrm{C}$ (48), might cause the small changes in the integrated PMMA amount.

Comparing the mechanical parameters determined for the varied PMMA composition, our data are in agreement with the observations in ref 38. The values for the stress peak and the tack energy of P(EHA-stat-20MMA) are increased by approximately a factor of 2 compared to those of the polymers consisting of $90 \%$ EHA and $10 \%$ MMA.

\section{CONCLUSION}

For the first time, the composition profiles perpendicular to the sample surface of PSA films consisting of a twocomponent statistical copolymer are successfully monitored. XRR as an investigation method turned out to be an ideal tool for this purpose. Different statistical copolymers were chosen to address the influence of the type of monomers and the sample age in this investigation.

Each probed sample shows a heterogeneous composition profile up to depths of approximately $100 \mathrm{~nm}$ (measured from the sample surface). For larger distances, the samples are homogeneous and the average monomer composition of the respective statistical copolymer is detected. For all polymers, the heterogeneous composition profile shows an oscillating behavior before it finally converges to the statistical monomer average.

Freshly prepared samples and aged samples differ in their composition profiles. The composition profile of freshly prepared samples is driven by solubility effects. As a consequence, for a given solvent, either the sticky or the glassy component of the statistical copolymer enriches at the surface irrespective of the majority component. The component being enriched at the sample surface is not changed by choosing another monomer ratio. The phase of the oscillation of the subsurface composition profile is determined by the type of monomer that is enriched at the surface.

Aging of the investigated PSA films arises from an internal reorganization process. The system minimizes its surface energy in a way that the component of the statistical copolymer with the smaller surface tension of the corresponding homopolymer enriches at the surface. Thus the reorganization is very strong in case the solubility driven morphology is different from the one with the minimized surface energy. Only in the case where solubility and surface tension favor the same component of the statistical copolymer at the surface of the adhesive film, the aging due to reorganization is minor. Furthermore, the effect of selfattraction of equal monomers is observed in regions that are not directly in contact with the sample surface.

Although the mechanical properties are mainly controlled by the bulk composition, the near-surface region contributes significantly to the adhesive properties. A strong influence arises from the different composition profiles due to the chosen chemical composition. However, the aging of the examined PSA does not result in a change of its performance because the content of the glassy component integrated over the punch roughness in the surface-near region does not vary.

In summary, this investigation points out important parameters that influence the molecular composition of the near-surface region of adhesive films. Moreover, the influence of the installed composition profile on the adhesive properties is investigated. This knowledge about the pos- 
sibility of a fine-tuning of the adhesive performance is beneficial for designing PSAs in special applications.

In addition, it can be concluded that for punches with a small surface roughness, the main contribution to the mechanical performance comes from the surface-near region and with decreasing punch roughness it has to be expected that the surface component is of increasing importance.

Acknowledgment. We thank B. Russ and P. Böni for their assistance in the XRR experiments and BASF SE for donating the polymer samples. The financial support by the Deutsche Forschungsgemeinschaft (DFG) in the projects MU 1487/6 and WI $3138 / 2$ is gratefully acknowledged.

\section{REFERENCES AND NOTES}

(1) Anderson, C. Ind. Eng. Chem. 1969, 61, 48-52.

(2) Sauer, B. B.; Gochanour, C. R.; Van Alsten, J. G. Macromolecules 1999, 32, 2739-2747

(3) Paiva, A.; Sheller, N.; Foster, M. D.; Crosby, A. J.; Shull, K. R. Macromolecules 2001, 34, 2269-2276.

(4) Severtson, S. J.; Wang, P. X.; Kroll, M. S. Ind. Eng. Chem. Res. 2002, 41, 5668-5675.

(5) Daoulas, K. C.; Theodorou, D. N.; Roos, A.; Creton, C. Macromolecules 2004, 37, 5093-5109.

(6) Müller-Buschbaum, P.; Ittner, T.; Petry, W. Europhys. Lett. 2004, 66,513

(7) González, I.; Leiza, J. R.; Asua, M. J. Macromolecules 2006, 39, 5015-5020

(8) Jeusette, M.; Leclère, P.; Lazzaroni, R.; Simal, F.; Vaneecke, J.; Lardot, T.; Roose, P. Macromolecules 2007, 40, 1055-1065.

(9) Müller-Buschbaum, P.; Ittner, T.; Maurer, E.; Körstgens, V.; Petry, W. Macromol. Mater. Eng. 2007, 292, 793

(10) Maji, P. K.; Guchhait, P. K.; Bhowmick, A. K. ACS Appl. Mater. Interfaces 2009, 1, 289-300

(11) Wang, T.; Canetta, E.; Weerakkody, T. G.; Keddie, J. L.; Rivas, U. ACS Appl. Mater. Interfaces 2009, 1, 631-639.

(12) Lindner, A.; Maevis, T.; Brummer, R.; Lühmann, B.; Creton, C. Langmuir 2004, 20, 9156 -9169.

(13) Girard-Reydet, E.; Oslanec, R.; Whitten, P.; Brown, H. R. Langmuir 2004, 20, 708-713.

(14) Flanigan, C. M.; Crosby, A. J.; Shull, K. R. Macromolecules 1999 32, $7251-7262$.

(15) Xu, G. H.; Dong, J.; Zhang, J.; Severtson, J. S.; Houtman, C. J.; Gwin, L. E. J. Phys. Chem. B 2008, 112, 11907-11914.

(16) Jovanović, R.; Dubé, M. A. Ind. Eng. Chem. Res. 2005, 44, $6668-$ 6675.

(17) Müller-Buschbaum, P.; Gutmann, J. S.; Stamm, M. Macromolecules 2000, 33, 4886-4895

(18) Schwarz, S. A.; Wilkens, B. J.; Pudensi, M. A. A.; Rafailovich, M. H.; Sokolov, J.; Zhao, W.; Zheng, X.; Russel, T. P.; Jones, R. A. L. Mol. Phys. 1992, 76, 937-950.

(19) Jones, R. A. L.; Kramer, E. J.; Rafailovich, M. H.; Sokolov, J.; Schwarz, S. A. Phys. Rev. Lett. 1989, 62, 281-283.

(20) El-Mabrouk, K.; Belaiche, M.; Bousmina, M.J. Colloid Interface Sci. 2007, 306, 354-367.

(21) Busch, P.; Posselt, D.; Smiglies, D.-M.; Rauscher, M.; Papadakis, C. M. Macromolecules 2007, 40, 630-640

(22) Busch, P.; Posselt, D.; Smiglies, D.-M.; Rheinländer, B.; Kremer, F.; Papadakis, C. M. Macromolecules 2003, 36, 8717-8727.

(23) Anastasiadis, S. H.; Russel, T. P.; Satija, S. K.; Majkrzak, C. F. Phys. Rev. Lett. 1989, 62, 1852-1855.

(24) Coutandin, J.; Ehlich, D.; Sillescu, D.; Chin-Hsien, W. Macromolecules 1985, 350, 589-590.

(25) Lauter-Pasyuk, V.; Lauter, H.; Gordeev, G.; Müller-Buschbaum, P.;
Toperverg, B. P.; Petry, W.; Jerenkov, M.; Aksenov, V. Physica B 2004, 350, e939-e942

(26) Ge, S.; Guo, L.; Rafailovich, M. H.; Sokolov, J.; Peiffer, D. G.; Schwarz, S. A.; Colby, R. H.; Dozier, W. D. Langmuir 1999, 15, $2911-2915$.

(27) Falsafi, A.; Tirrel, M.; Pocius, A. V. Langmuir 2000, 16, 1816 1824

(28) Donley, J. P.; Fredrickson, G. H. Macromolecules 1994, 27, 458 467.

(29) Klos, J. S.; Romeis, D.; Sommer, J.-U.J. Chem. Phys. 2010, 132, 024907.

(30) Amouroux, N.; Petit, J.; Léger, L. Langmuir 2001, 17, 6510-6517.

(31) Zhang Newby, B.-m.; Chaudhury, M. K. Langmuir 1998, 14, 48654872 .

(32) Zhang Newby, B.-m.; Chaudhury, M. K.; Brown, H. R. Science 1995, 269, 1407-1409

(33) Zhang Newby, B.-m.; Chaudhury, M. K. Langmuir 1997, 13, $1805-$ 1809 .

(34) Deplace, F.; Carelli, C.; Langenfeld, A.; Rabjohns, M. A.; Foster, A. B.; Lovell, P. A.; Creton, C. ACS Appl. Mater. Interfaces 2009, 1 (9), 2021-2029.

(35) Kim, J. K.; Ryu, D. Y.; Lee, K.-H. Polymer 2000, 41, 5195-5205.

(36) Ryu, D. Y.; Kim, J. K. Polymer 2000, 41, 5207-5218.

(37) Kano, Y.; Ushiki, H.; Akiyama, S. J. Adhes. 1993, 43, 223-227.

(38) Aymonier, A.; Papon, E.; Villenave, J.-J.; Tordjeman, Ph.; Pirri, R.; Gérard, P. Chem. Mater. 2001, 13, 2562-2566.

(39) Aymonier, A.; Leclercq, D.; Tordjeman, P.; Papon, E.; Villenave, J.-J.J. Appl. Polym. Sci. 2003, 89, 2749-2756

(40) Laureau, C.; Vicente, M.; Barandiaran, M. J.; Leiza, J. R.; Asua, J. M. J. Appl. Polym. Sci. 2001, 81, 1258-1265.

(41) Parratt, L. G. Phys. Rev. 1954, 95, 359-369.

(42) Born, M.; Wolf, E. Principles of Optics, 2nd ed.; Pergamon Press: Oxford, 1964.

(43) James, R. W. The Optical Principles of the Diffraction of X-rays; OxBow Press: Woodbridge, CT, 1962.

(44) Müller-Buschbaum, P. Euro. Phys. J. E 2003, 12, 443

(45) Braun, C. Parratt32, version 1.6; HMI Berlin: Berlin, 2002.

(46) Lekner, J. Theory of Reflection; Martinus Nijhoff Publishers: Dodrecht, The Netherlands, 1987.

(47) Feidenhans'l, R. Surf. Sci. Rep. 1989, 10, 105-188.

(48) Bandrup, J.; Immergut, E. H.; Grulke, E. A.; Bloch, D. Polymer Handbook, fourth ed.; Wiley-Interscience: Hoboken, NJ, 1999.

(49) Walheim, S.; Böltau, M.; Mlynek, J.; Krausch, G.; Steiner, U. Macromolecules 1997, 30, 4995-5003.

(50) Ngwa, W.; Wannemacher, R.; Grill, W.; Serghei, A.; Kremer, F.; Kundu, T. Macromolecules 2004, 37, 1691-1692.

(51) Zhuang, H.; Gardella, J. A. Macromolecules 1997, 30, 3632-3639.

(52) Elbs, H.; Fukunaga, K.; Stadler, R.; Sauer, G.; Magerle, R.; Krausch, G. Macromolecules 1999, 32, 1204-1211

(53) Perlich, J.; Körstgens, V.; Metwalli, E.; Schulz, L.; Georgii, R.; Müller-Buschbaum, P. Macromolecules 2009, 42, 337-344.

(54) da Rocha, S. R. P.; Harrison, K. L.; Johnston, K. P. Langmuir 1999. $15,419-428$

(55) Kwok, D. Y.; Leung, A.; Lam, C. N. C.; Li, A.; Wu, R.; Neumann, A. W. J. Colloid Interface Sci. 1998, 206, 44-51

(56) Chen, W.; Lee, S.; Ho, B. J. Polym. Res. 1998, 5, 187-191.

(57) Frank, B.; Gast, A. P.; Russell, T. P.; Brown, H. R.; Hawker, C. Macromolecules 1996, 29, 6531-6534.

(58) Sharma, A.; Reiter, G. J. Colloid Interface Sci. 1996, 178, 383-399.

(59) Lakrout, H.; Creton, C.; Dongchan, A.; Shull, K. R. Macromolecules 2001, 34, 7448-7458.

(60) O'Connor, A. E.; Willenbacher, N. Int. J. Adhes. Adhes. 2004, 24, 335-346.

(61) Peykova, Y.; Guriyanova, S.; Lebedeva, O. V.; Diethert, A.; MüllerBuschbaum, P.; Willenbacher, N. Int. J. Adhes. Adhes. 2010, 30, $245-254$

AM100322J 